\title{
A CHARACTERIZATION OF COMPLETE METRIC SPACES
}

\author{
FRANCIS SULLIVAN
}

Abstract. A general formulation of the completeness argument used in the Bishop-Phelps Theorem and many other places has been given by Ekeland. It is shown that Ekeland's formulation characterizes complete metric spaces.

A central idea in the proof of the Bishop-Phelps Theorem is the use of norm completeness and a partial ordering to produce a point where a linear functional attains its supremum on a closed bounded convex set. In fact, this completeness technique is useful in many situations as has been described in the surveys of Phelps [7] and Brezis-Browder [1]. Recently Ekeland [3] has given a very general formulation of this technique and has applied it to a wide variety of problems [4]. In the present note we show that Ekeland's formulation is actually equivalent to completeness for metric spaces.

Ekeland's Theorem may be stated as follows:

TheOREM 1. Let $(M, d)$ be a complete metric space, and $F: M \rightarrow \mathbf{R} \cup\{+\infty\} a$ lower semicontinuous function, $F \neq+\infty$, bounded from below. Let $\varepsilon>0$ be given and $a$ point $u \in M$ such that

$$
F(u) \leqslant \inf _{M} F+\varepsilon .
$$

Then there exists a point $v \in M$ such that

(i) $F(v) \leqslant F(u)$.

(ii) $d(u, v) \leqslant 1$.

(iii) For all $w \neq v, F(w)+\varepsilon d(v, w)>F(v)$.

This result characterizes completeness of $M$ in the following sense.

THEOREM 2. Let $(M, d)$ be a metric space. Then $M$ is complete if and only if for every continuous $F: M \rightarrow \mathbf{R} \cup\{+\infty\}, F \neq+\infty$, bounded from below and every $\varepsilon>0$ there is a point $v \in M$ satisfying

(i) $F(v) \leqslant \inf _{M} F+\varepsilon$ and (iii) above.

Proof. The "only if" direction is immediate from Theorem 1. For the converse assume that $(M, d)$ is an arbitrary metric space satisfying the hypotheses. Let $\left(y_{n}\right) \subseteq M$ be a Cauchy sequence and consider the function $F: M \rightarrow \mathbf{R}$ given by

$$
F(x)=\lim _{n} d\left(y_{n}, x\right) \text {. }
$$

Received by the editors November 20, 1980.

AMS (MOS) subject classifications (1970). Primary 26A54, 34A05, 46B99.

Key words and phrases. Bishop-Phelps Theorem, completeness.

(c) 1981 American Mathematical Society 0002-9939/81/0000-0475/\$01.50 
The function $F$ is continuous and, since $\left(y_{n}\right)$ is Cauchy, inf $F=0$. To show completeness we produce a $v \in M$ such that $F(v)=0$.

Choose any $0<\varepsilon<1$. Now, from (i)' and (iii), there is a $v \in M$ with $F(v)<\varepsilon$ and $F(w)+\varepsilon d(w, v)>F(v)$ for all $w \neq v$. From the definition of $F$ and the fact that $\left(y_{n}\right)$ is Cauchy we can take $w=y_{p}$ for $p$ large such that $F(w)$ is arbitrarily small and $d(w, v) \leqslant \varepsilon+\eta$ for any $\eta>0$, because $F(v)<\varepsilon$. Using condition (iii) we get that, in fact, $F(v) \leqslant \varepsilon^{2}$. Repeating the argument we may conclude that $F(v) \leqslant \varepsilon^{n}$ for all $n \geqslant 1$ and so $F(v)=0$ as required. Q.E.D.

REMARK. Caristi [2] and Kirk [5] have studied connections between completeness and fixed-point theorems. In [6] Kirk proves that a fixed point property related to Ekeland's formulation also characterizes complete metric spaces.

\section{REFERENCES}

1. H. Brezis and F. Browder, A general ordering principle in nonlinear functional analysis, Adv. in Math. 21 (1976), 355-364.

2. J. Caristi, Fixed point theorems for mappings satisfying inwardness conditions, Trans. Amer. Math. Soc. 215 (1976), 241-251.

3. I. Ekeland, On the variational principle, J. Math. Anal. Appl. 47 (1974), 324-353.

4. __ Nonconvex minimization problems, Bull. Amer. Math. Soc. (N. S.) 1 (1979), 443-474.

5. W. A. Kirk, Caristi's fixed point theorem and the theory of normal solvability, (Proc. Sem. Fixed Point Theory and its Appl., Dalhousie University, 1975), Academic Press, New York, 1976.

6. , Caristi's fixed point theorem and metric convexity, Colloq. Math. 36 (1976), 81-86.

7. R. R. Phelps, Support cones in Banach spaces and their applications, Adv. in Math. 13 (1974), 1-19.

Department of Mathematics, Catholic University of America, Washington, D.C. 20064 (Current address)

Mathematisch InstituUt, Katholizke Universitert, Tokrnooiveld, 6525 ED Nimmgen, The NetHERLANDS 